Fourier spectral methods for fractional-in-space reaction-diffusion equations

by

\author{
Alfonso Bueno-Orovio \\ David Kay \\ Kevin Burrage
}





\title{
Fourier spectral methods for fractional-in-space reaction-diffusion equations
}

\author{
Alfonso Bueno-Orovio ${ }^{\mathrm{a}, \mathrm{b}, *}$, David Kay ${ }^{\mathrm{b}}$, Kevin Burrage ${ }^{\mathrm{b}, \mathrm{c}}$ \\ ${ }^{a}$ Oxford Centre for Collaborative Applied Mathematics, University of Oxford, \\ Oxford OX1 3LB, United Kingdom \\ ${ }^{b}$ Computational Biology Group, Department of Computer Science, University of Oxford, \\ Oxford OX1 3QD, United Kingdom \\ ${ }^{c}$ School of Mathematical Sciences, Queensland University of Technology, \\ Brisbane 4001, Australia
}

\begin{abstract}
Fractional differential equations are becoming increasingly used as a powerful modelling approach for understanding the many aspects of nonlocality and spatial heterogeneity. However, the numerical approximation of these models is computationally demanding and imposes a number of computational constraints. In this paper, we introduce Fourier spectral methods as an attractive and easy-to-code alternative for the integration of fractional-in-space reactiondiffusion equations. The main advantages of the proposed schemes is that they yield a fully diagonal representation of the fractional operator, with increased accuracy and efficiency when compared to low-order counterparts, and a completely straightforward extension to two and three spatial dimensions. Our approach is show-cased by solving several problems of practical interest, including the fractional Allen-Cahn, FitzHugh-Nagumo and Gray-Scott models, together with an analysis of the properties of these systems in terms of the fractional power of the underlying Laplacian operator.
\end{abstract}

Keywords: Fractional calculus, fractional Laplacian, spectral methods, reaction-diffusion equations

2010 MSC: 35R11, 65M70, 34L10, 65T50, 35K57

\section{Introduction}

Fractional differential equations are becoming increasingly used as a modelling tool for diffusive processes associated with sub-diffusion (fractional in time), super-diffusion (fractional in space) or both, and have a long history in,

\footnotetext{
${ }^{*}$ Corresponding author

Email addresses: alfonso.bueno@maths.ox.ac.uk (Alfonso Bueno-Orovio), david.kay@cs.ox.ac.uk (David Kay), kevin.burrage@cs.ox.ac.uk (Kevin Burrage)
} 
for example, physics, finance, mathematical biology and hydrology. In water resources, fractional models have been used to describe chemical and contaminant transport in heterogeneous aquifers $[1,2,3]$. In finance, they have been used because of the relationship with certain option pricing mechanisms and heavy tailed stochastic processes [4]. More recently, fractional models of the BlochTorrey equation have been used in magnetic resonance [5]. In this paper we will only consider super-diffusion effects (space-fractional models) in spatially extended structures, such as those possessing spatial connectivity, where the movement of particles may thus be facilitated within a certain scale.

In this context, the spatial complexity of the environment imposes geometric constraints on the transport processes on all length scales, which can be interpreted as temporal correlations on all time scales. Non-homogeneities of the medium may fundamentally alter the laws of Markov diffusion, leading to long range fluxes, and non-Gaussian, heavy tailed profiles [6, 7], and these motions may no longer obey Fick's Law [8]. It is in this setting that fractional models can offer insights that traditional approaches do not offer.

A space-fractional diffusion equation can be derived by replacing Fick's Law for the flux $V$ (the rate at which mass is transported through an unit area against the concentration gradient) by its fractional counterpart (cf. Meerschaert et al. $[9])$ :

$$
V=-\mathbf{K} \nabla^{\beta} u, \quad 0<\beta \leq 1,
$$

where $\mathbf{K}$ is the conductivity or diffusion tensor, and $\nabla^{\beta}=\left(\frac{\partial^{\beta}}{\partial x^{\beta}}, \frac{\partial^{\beta}}{\partial y^{\beta}}, \frac{\partial^{\beta}}{\partial z^{\beta}}\right)^{T}$ is the Riemann-Louiville fractional gradient, where

$$
\frac{\partial^{\beta}}{\partial x^{\beta}} u(x, y, z)=\frac{1}{\Gamma(1-\beta)} \frac{\partial}{\partial x} \int_{0}^{x} \frac{u(s, y, z)}{(x-s)^{\beta}} d s,
$$

with similar expressions for $\frac{\partial^{\beta}}{\partial y^{\beta}}$ and $\frac{\partial^{\beta}}{\partial z^{\beta}}$ [10]. The fractional Fick's Law (1) naturally implies spatial and temporal nonlocality, and can be derived from rigorous approaches using spatial averaging theorems and measurable functions [11]. Combining this with a conservation of mass equation for the concentration of particles $u(\mathbf{x}, t)$

$$
\partial_{t} u=-\nabla \cdot V
$$

finally leads to

$$
\partial_{t} u=-\nabla \cdot\left(-\mathbf{K} \nabla^{\beta} u\right) .
$$

Equivalently, in the isotropic setting [12] the space fractional reaction-diffusion equation can be written as

$$
\partial_{t} u=-K(-\Delta)^{\alpha / 2} u+f(u, t), \quad 1<\alpha \leq 2,
$$

where $(-\Delta)^{\alpha / 2}$ is the fractional Laplacian operator.

A standard approach for solving problems of the form (5) is to apply a finite difference, finite element or finite volume discretisation of the fractional operator, and then use a semi-implicit Euler formulation for the time evolution of 
the solution. This requires the solution of a linear system of equations at each time step, whose left-hand-side matrix has a fractional power. Various authors such as Meerschaert et al. [13], Roop [14], Ilic et al., [15], Liu et al. [16] and Pang et al. [17] have considered the numerical solution of such problems using various discretisations, but most of these approaches either do not scale well or their scalability has not been demonstrated. Very recently, two approaches have been developed that use Krylov approaches [18] or fast numerical integration in conjunction with effective preconditioners [19] -see Section 2.1 for more details - that allow for problems in two or three spatial dimensions to be tackled. However, even these latter approaches do not scale perfectly as the spatial dimension increases to three and their effectiveness still depends on the mesh discretisation.

In this paper we introduce Fourier spectral methods as an efficient alternative approach to solving fractional reaction-diffusion problems in the form of (5). The main advantage of this approach is that it gives a full diagonal representation of the fractional operator, being able to achieve spectral convergence regardless of the fractional power in the problem. An additional advantage is that the application to two and three spatial dimensions is essentially the same as the one dimensional problem.

The outline of the paper is as follows. In Section 2 we discuss different numerical methods for the solution of problems with non-local diffusion processes. Section 3 gives the main elements of our spectral approach and presents some convergence analysis for different types of initial and boundary conditions. In Section 4 we present the applicability of these ideas to a number of important problems, involving metastability (the Allen-Cahn equation), excitable media (the FitzHugh-Nagumo model) and pattern formation in two and three dimensional space (the Gray-Scott model). Finally, Section 5 offers some conclusions and thoughts for future work.

\section{Numerical approaches for fractional diffusion}

Several numerical approaches have been suggested in the literature to overcome the nonlocal restrictions of space fractional operators. A brief survey of such methods is presented in this section.

\subsection{Finite element methods}

The main advantage of using a finite element approach is the flexibility the method offers. In particular, the ability to model complex geometries and to improve approximations by using adaptive local refinement. The main hurdle to overcome is the non-local nature of the fractional operator and thus a straight application of finite elements would lead to large dense matrices. Even the construction off such matrices presents difficulties, especially in efficiency, see, for example, [14].

One choice is to truncate the kernel, so that local interactions are only considered. When using this approach it is not a trivial exercise to obtain reliable 
approximations. Furthermore, to obtain optimal convergence would require the radius of truncation to increase, leading back to the original dense matrix structure. A second choice is to produce the standard discrete finite element Laplacian operator, $\mathbf{A}$, and use a partial diagonalisation of this matrix. This approach will suffer from the same issues as the first one. Finally, in the case of the fractional Laplacian, take the matrix representation, $\mathbf{A}$, of the Laplacian and raise it to the desired fractional power. An alternative approach to the latter method, known as the Matrix Transfer Technique (MTT), is to compute the direct product of the function of a matrix times a vector. This avoids raising the matrix $\mathbf{A}$ to the fractional power, thus retaining the sparse structure of the finite element approximation of the operator. Yang et al. [18] have solved the time-space fractional diffusion equation in two spatial dimensions with homogeneous Dirichlet boundary conditions using the MTT either by a preconditioned Lanczos (symmetric) or a M-Lanczos (non-symmetric) technique. Recently, Burrage et al. [19] have developed a robust, efficient MTT approach that can be equally applicable to fractional-in-space problems in two or three spatial dimensions on structured and unstructured grids. They considered three techniques: the contour integral method, Extended Krylov subspace methods, and the pre-assigned poles and interpolation nodes method, and found preconditioners that allow almost mesh independent convergence and which thus scale to solving three dimensional problems.

\subsection{Finite difference methods}

Finite differences are typically defined on well structured grids. In the case of the fractional operator two approaches may be taken. The first is to apply the fractional power to the finite difference Laplacian matrix using the techniques mentioned above. Alternatively a finite difference formula on tensor grids using a shifted Grünwald discretisation may be applied [13, 15, 16, 20]. When applied in two and three space dimensions this approach leads to relatively sparse, well structured, positive definite matrices. The solution of these linear systems can be approximated efficiently using a combination of multigrid and conjugate gradient methods, see $[17,21]$. As well as relying on having simple geometries, finite difference approximations are not capable of exploiting solutions with high regularity.

\subsection{Spectral methods}

Despite their higher order of convergence when compared to low order stencils and being in nature nonlocal, little use has been done of spectral methods for the solution of fractional-in-space equations. $\mathrm{Li}$ and $\mathrm{Xu}$ [22] have considered a spectral approach for the weak solution of the space-time fractional diffusion equation. Khader [23] proposes a Chebyshev Galerkin method for the discretisation of the fractional diffusion equation where the spatial derivatives are considered in the Caputo sense, similar to the results of $\mathrm{Li}$ and $\mathrm{Xu}$ [24] for the time-fractional diffusion equation. Hanert [25] also has considered the use of a Chebyshev spectral element method for the numerical solution of the 
fractional Riemann-Louiville advection-diffusion equation for tracer transport. However, all previous works were restricted to one-dimensional simulations, and to our knowledge there is no rigorous study on the application of Fourier spectral methods to fractional-in-space reaction-diffusion equations. This will be the main contribution of this paper. We will show that fractional-in-space reactiondiffusion equations, with simple geometries and boundary conditions, can be solved efficiently and accurately using this approach.

\section{Fourier spectral method for fractional diffusion}

Spectral decomposition plays a central role in the interpretation of the fractional Laplacian - see [15] and [20]. Suppose the Laplacian $(-\Delta)$ has a complete set of orthonormal eigenfunctions $\left\{\varphi_{j}\right\}$ satisfying standard boundary conditions on a bounded region $\mathcal{D} \subset \mathbb{R}^{d}$, with corresponding eigenvalues $\lambda_{j}$, i.e., $(-\Delta) \varphi_{j}=\lambda_{j} \varphi_{j}$ on $\mathcal{D}$, and let

$$
\mathcal{U}_{\alpha}:=\left\{u=\sum_{j=0}^{\infty} \hat{u}_{j} \varphi_{j}, \hat{u}_{j}=\left\langle u, \varphi_{j}\right\rangle, \sum_{j=0}^{\infty}\left|\hat{u}_{j}\right|^{2}\left|\lambda_{j}\right|^{\alpha / 2}<\infty, 1<\alpha \leq 2\right\} .
$$

Then, for any $u \in \mathcal{U}_{\alpha}$, it holds

$$
(-\Delta)^{\alpha / 2} u=\sum_{j=0}^{\infty} \hat{u}_{j} \lambda_{j}^{\alpha / 2} \varphi_{j}
$$

Therefore, $(-\Delta)^{\alpha / 2}$ has the same interpretation as $(-\Delta)$ in terms of its spectral decomposition. Furthermore, the former result suggests that a spectral approach may be feasible for solving problems in the form of (5).

\subsection{Space discretisation}

In order to present the bases of the method, let us consider for simplicity the one-dimensional space fractional heat equation in the absence of source term

$$
\partial_{t} u=-K(-\Delta)^{\alpha / 2} u
$$

subject to $u(x, 0)=u_{0}(x)$ and any of the standard homogeneous boundary conditions in $x \in[a, b]$. By using (6)-(7), we can easily derive the analytical solution of (8) as

$$
u(x, t)=\sum_{j=0}^{\infty} \hat{u}_{j}(t) \varphi_{j}(x)=\sum_{j=0}^{\infty} \hat{u}_{j}(0) e^{-K \lambda_{j}^{\alpha / 2} t} \varphi_{j}(x)
$$

with $\hat{u}_{j}(0)=\left\langle u_{0}(x), \varphi_{j}(x)\right\rangle$. Eigenfunctions and eigenvalues will depend on the specified boundary conditions: $\lambda_{j}=\left(\frac{(j+1) \pi}{L}\right)^{2}, \varphi_{j}=\sqrt{\frac{2}{L}} \sin \left(\frac{(j+1) \pi(x-a)}{L}\right)$ for 
Code 1: Fractional heat equation (homogeneous Dirichlet boundary conditions).

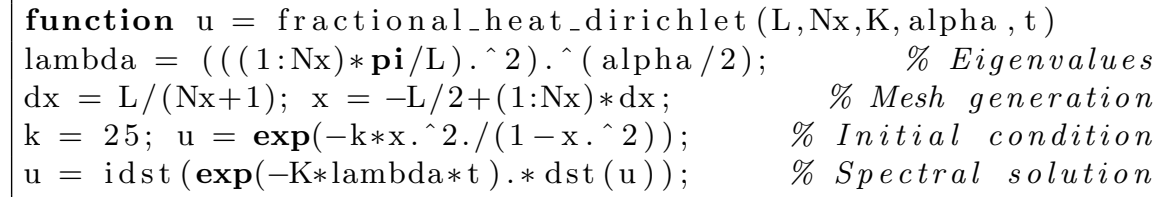

Code 2: Fractional heat equation (homogeneous Neumann boundary conditions).

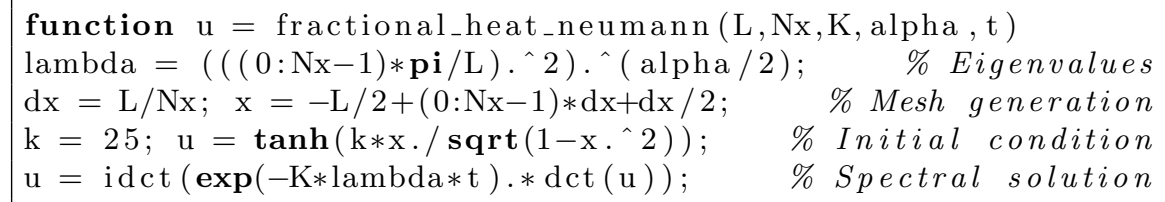

homogeneous Dirichlet; $\lambda_{j}=\left(\frac{j \pi}{L}\right)^{2}, \varphi_{j}=\sqrt{\frac{2}{L}} \cos \left(\frac{j \pi(x-a)}{L}\right)$ for homogeneous Neumann; and $\lambda_{j}=\left(\frac{2 \pi j}{L}\right)^{2}, \varphi_{j}=e^{i \frac{2 \pi j}{L}(x-a)}$ for periodic ones, where $L=b-a$.

Fourier spectral methods represent the truncated series expansion of (9) when a finite number of orthonormal trigonometric eigenfunctions $\left\{\varphi_{j}\right\}$ (equal to the number of discretisation points) are considered

$$
\hat{u}(x, t) \approx \sum_{j=0}^{N-1} \hat{u}_{j}(t) \varphi_{j}(x)=\sum_{j=0}^{N-1} \hat{u}_{j}(0) e^{-K \lambda_{j}^{\alpha / 2} t} \varphi_{j}(x) .
$$

For each of the specified types of boundary data, coefficients $\hat{u}_{j}$ in (10), as well as the inverse reconstruction of $u$ in physical space, can be efficiently computed by fast and robust existing algorithms (direct and inverse Discrete Sine/Cosine/ Fourier Transforms, see $[26,27])$. To illustrate the ease of application of the approach, the 5 -lines of MATLAB Codes 1 and 2 exemplify the numerical solution of equation (8) in $x \in[-L / 2, L / 2]$, subject to homogeneous Dirichlet and Neumann boundary conditions, respectively. Note the differences in mesh generation due to odd/even restrictions imposed by the corresponding basis functions.

\subsection{Convergence in space}

Equivalently, the solution of (8) using a finite differences or finite elements matrix-based approach can be approximated as

$$
\hat{u}(x, t) \approx \mathbf{Q} \operatorname{diag}\left\{e^{-K \lambda_{0}^{\alpha / 2} t}, e^{-K \lambda_{1}^{\alpha / 2} t}, \ldots, e^{-K \lambda_{N-1}^{\alpha / 2} t}\right\} \mathbf{Q}^{-1} \mathbf{u}_{0},
$$

where $\mathbf{Q}$ represents the matrix of corresponding eigenvectors and $\mathbf{u}$ denotes the vector of node values of $u$. Since both (10) and (11) are exact in time, all of the error in both schemes is associated with the spatial discretisation, so we 


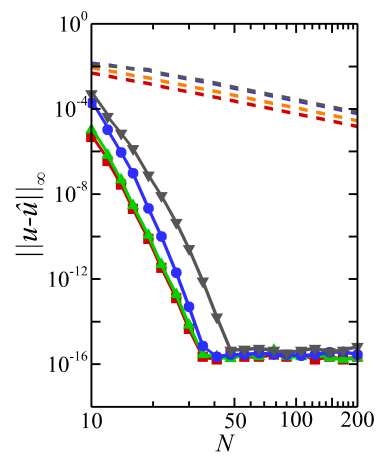

(a) $u_{0}(x)=e^{-k \frac{x^{2}}{1-x^{2}}}$

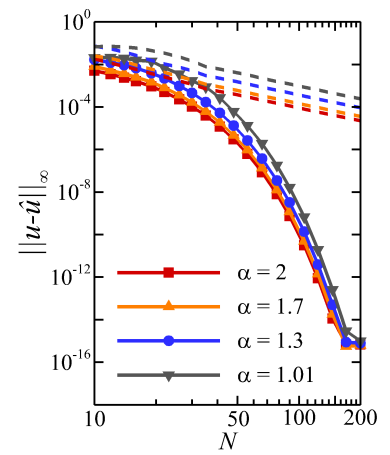

(b) $u_{0}(x)=\tanh \left(\frac{k x}{\sqrt{1-x^{2}}}\right)$
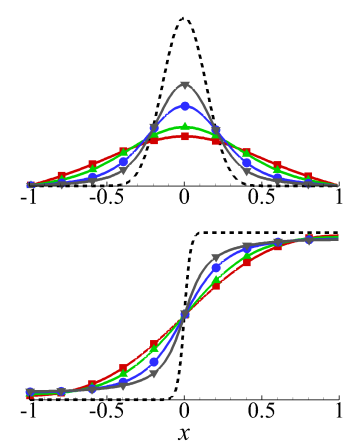

(c) Evolution of initial data

Figure 1: Convergence results for the fractional heat equation (8) for Fourier (solid lines) and finite difference (dashed lines) methods. A value of $k=25$ was used in both cases. Right panel illustrates evolution of initial data (dashed black lines) at $t=0.1$ for varying $\alpha$.

can use this simple example to study the convergence of the two schemes in the numerical approximation of (8) for varying values of the fractional power $\alpha$.

Convergence results in the $\ell^{\infty}$-norm are presented in Figure 1 for the Fourier and finite differences approximations of (8) on $x \in[-1,1]$ at $t=0.1$, with $K=1$. Two different initial conditions were considered: a smooth Gaussian profile, subject to homogeneous Dirichlet conditions (Fig. 1(a)); and a sigmoid exhibiting sharper gradients, with homogeneous Neumann data (Fig. 1(b)). Reference solutions were calculated by evaluating (9) with $2^{12}$ Fourier modes, with coefficients $\hat{u}_{j}$ computed by adaptive Gauss-Kronrod quadrature. In both situations, the Fourier approach is able to achieve spectral convergence up to machine precision regardless of $\alpha$, whereas the finite differences solutions show the standard $\mathcal{O}\left(N^{-2}\right)$ accuracy of these schemes. As expected, in the presence of initial data displaying steeper transitions, a larger number of discretisation points is required in the Fourier method to significantly reduce the approximation error. Figure 1(c) shows the effect of the fractional order in space for this problem, reflecting the slower rate of diffusion for $1<\alpha<2$.

Execution times are given in Table 1 for comparison between both methods, showing a much better performance of the Fourier method, especially for larger $N$. All computations were performed on an i5 Intel $2.3 \mathrm{GHz}$ laptop in MATLAB 7.6.

\subsection{Time discretisation}

For reaction-diffusion problems in the form of (5), we consider a backward Euler discretisation of the time derivative, where in each time step $\left[t_{n}, t_{n+1}\right]$ the nonlinear term is treated using the following fixed point iteration: given $u^{n}$, 


\begin{tabular}{|c|c|c|c|c|c|}
\hline$N$ & 32 & 64 & 128 & 256 & 512 \\
\hline Fourier & $1.4149 \mathrm{e}-4$ & $1.7433 \mathrm{e}-4$ & $2.2572 \mathrm{e}-4$ & $2.8651 \mathrm{e}-4$ & $3.8439 \mathrm{e}-4$ \\
\hline Finite Differences & $6.3810 \mathrm{e}-4$ & $2.1640 \mathrm{e}-3$ & $9.4125 \mathrm{e}-3$ & $5.7550 \mathrm{e}-2$ & $3.8384 \mathrm{e}-1$ \\
\hline Ratio & 4.51 & 12.41 & 41.70 & 200.87 & 998.57 \\
\hline
\end{tabular}

Table 1: Timing results (seconds) for solving equation (8) in one space dimension for various values of discretisation. Results are independent on $\alpha$.

define $u^{n+1,0}:=u^{n}$, and for $m=1,2, \ldots, M$ find $u^{n+1, m}$ such that

$$
\frac{u^{n+1, m}-u^{n}}{\Delta t}=-K(-\Delta)^{\alpha / 2} u^{n+1, m}+f\left(u^{n+1, m-1}, t^{n+1}\right),
$$

where $M$ is to be chosen. Clearly, $M=1$ leads to the fully explicit treatment of the nonlinear term, and for sufficiently large $M$, provided the iteration is converging, the method is fully implicit. The orthogonality of the basis functions implies that each of the Fourier coefficients evolves independently to the others, so for the $j$-th Fourier mode the time-space discretisation (12) simply becomes

$$
\hat{u}_{j}^{n+1, m}=\frac{1}{1+K \lambda_{j}^{\alpha / 2} \Delta t}\left[\hat{u}_{j}^{n}+\Delta t \hat{f}_{j}\left(u^{n+1, m-1}, t^{n+1}\right)\right],
$$

where $\hat{f}_{j}$ is the $j$-th Fourier coefficient of the source term. Note that (13) is fully diagonal, thus requiring no preconditioning, and that it also avoids associated numerical challenges for the treatment of singular Laplacians (containing the eigenvalue $\lambda_{j}=0$ ), as in the case of periodic or homogeneous Neumann boundary conditions [19].

\subsection{Convergence in time}

The Matlab Code 3 exemplifies the use of the above presented time-space stencil (13) for the numerical solution of (5), with

$$
\begin{aligned}
f(u, t)= & \frac{K}{4} t^{\alpha}\left\{3\left[1+(2 \pi)^{\alpha}\right] \sin (2 \pi x)-\left[1+(6 \pi)^{\alpha}\right] \sin (6 \pi x)\right\}+ \\
& \alpha t^{\alpha-1} \sin ^{3}(2 \pi x)-K u
\end{aligned}
$$

in $x \in[0,1]$, subject to $u(0, t)=u(1, t)=0, u(x, 0)=0$, and $K=10$. The exact solution to this problem is $u(x, t)=t^{\alpha} \sin ^{3}(2 \pi x)$, which can be verified applying Definition (7). Errors in the $\ell^{\infty}$-norm in the numerical solution at $t=1$ are listed in Table 2 for different time steps and number of fixed-point iterations, using $\alpha=1.5$ and $N=51$ discretisation points. As expected, for the implicit Euler method, the order of convergence in time for the scheme is $\mathcal{O}(\Delta t)$. Table 2 also indicates that the numerical error of the method is more controlled by the time resolution, $\Delta t$, than by the number of fixed-point iterations, $M$. 
Code 3: Fractional heat equation with source (homogeneous Dirichlet boundary conditions).

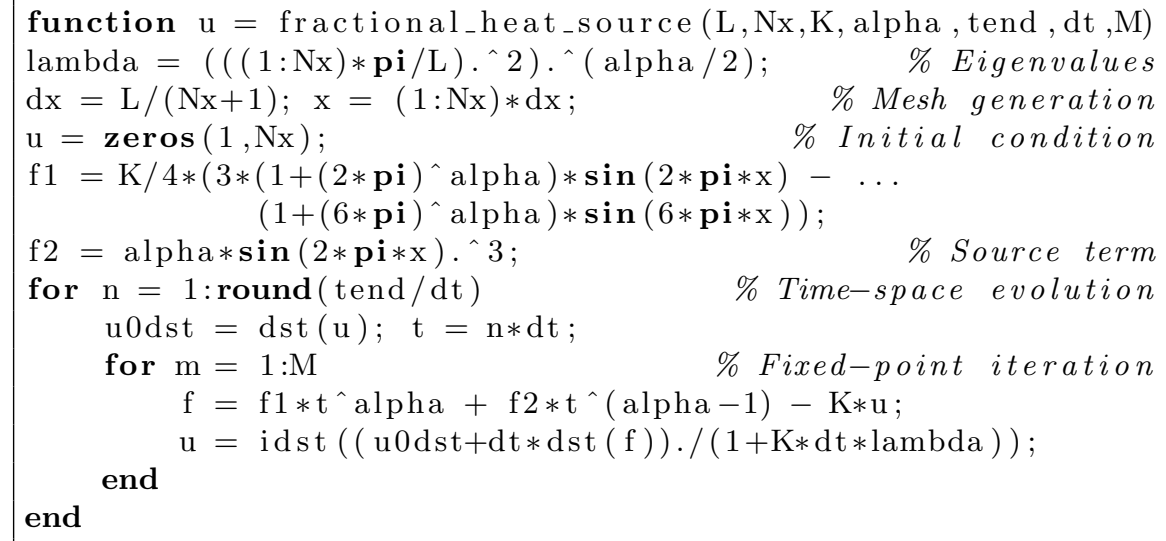

\begin{tabular}{|c|c|c|c|c|c|c|}
\hline$\Delta t$ & 0.1 & 0.05 & 0.025 & $1 \mathrm{e}-3$ & $5 \mathrm{e}-4$ & $2.5 \mathrm{e}-4$ \\
\hline$M=1$ & $7.1693 \mathrm{e}-3$ & $3.6247 \mathrm{e}-3$ & $1.8223 \mathrm{e}-3$ & $7.3271 \mathrm{e}-5$ & $3.6639 \mathrm{e}-5$ & $1.8321 \mathrm{e}-5$ \\
\hline$M=2$ & $2.3661 \mathrm{e}-4$ & $1.0937 \mathrm{e}-4$ & $4.4913 \mathrm{e}-5$ & $1.1893 \mathrm{e}-6$ & $7.3485 \mathrm{e}-7$ & $4.0667 \mathrm{e}-7$ \\
\hline$M=3$ & $2.0763 \mathrm{e}-4$ & $1.0176 \mathrm{e}-4$ & $4.9624 \mathrm{e}-5$ & $1.8029 \mathrm{e}-6$ & $8.9955 \mathrm{e}-7$ & $4.4948 \mathrm{e}-7$ \\
\hline$M=4$ & $1.8145 \mathrm{e}-4$ & $9.0026 \mathrm{e}-5$ & $4.4905 \mathrm{e}-5$ & $1.7977 \mathrm{e}-6$ & $8.9879 \mathrm{e}-7$ & $4.4938 \mathrm{e}-7$ \\
\hline
\end{tabular}

Table 2: Time convergence in the solution of the fractional heat equation with source term at $t=1(\alpha=1.5, N=51)$, subject to $u(0, t)=u(1, t)=0, u(x, 0)=0$, and $K=10$.

\section{Numerical examples}

The former results show that the global interpolant properties and the diagonal structure of the proposed Fourier spectral method enable the accurate and efficient simulation of fractional-in-space dynamical systems. In this section, we present numerical results of large-scale simulations of different reaction-diffusion models of general interest, with a level of spatial resolution unreported to date in fractional calculus computations in two and three dimensions. Due to their wide use in this type of models, we will concentrate here on the use of homogeneous Neumann boundary conditions, $\partial_{n} u=0$.

\subsection{Allen-Cahn equation - Metastability}

The Allen-Cahn equation with a quartic double well potential is a simple nonlinear reaction-diffusion model that arises in the study of formation and motion of phase boundaries. The fractional-in-space version of this equation takes the form

$$
\partial_{t} u=-K(-\Delta)^{\alpha / 2} u+u-u^{3}
$$

where $K$ is a small positive constant, and $\alpha=2$ represents the pure diffusion case. The steady states $u= \pm 1$ are attracting, and solutions tend to exhibit flat 
Code 4: Fractional Allen-Cahn equation (homogeneous Neumann boundary conditions).

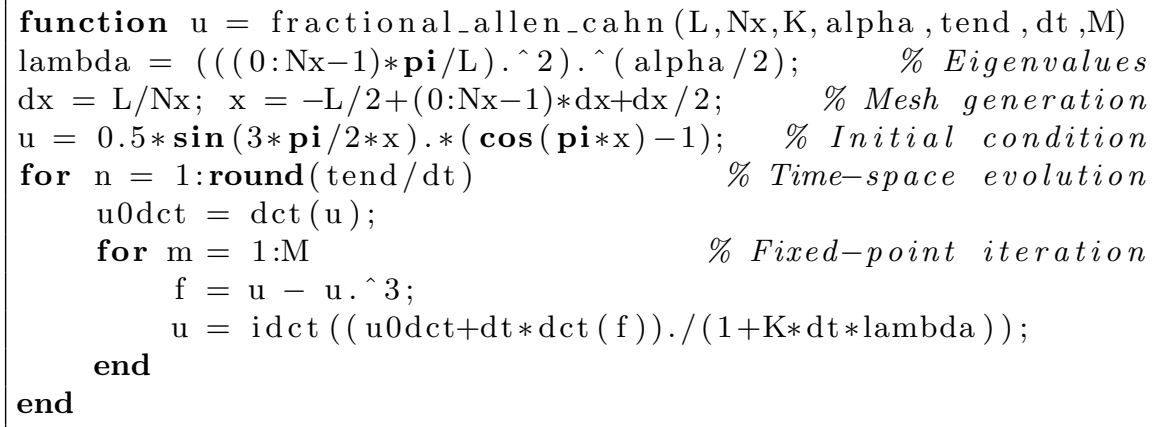

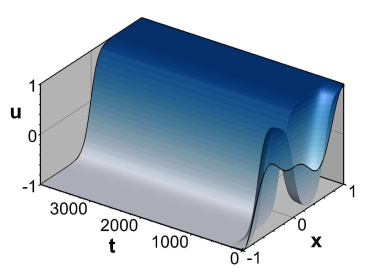

(a) $\alpha=2$

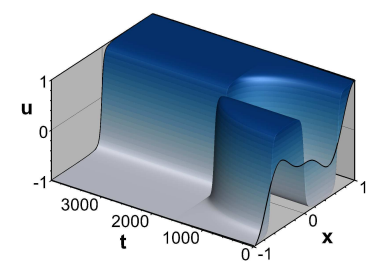

(b) $\alpha=1.3$

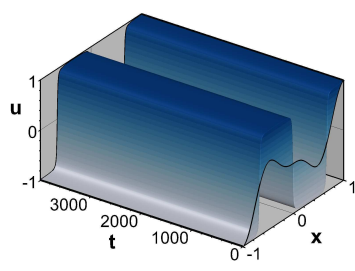

(c) $\alpha=1.01$

Figure 2: Metastability of solutions of the Allen-Cahn equation for varying $\alpha$.

areas around these two values separated by interfaces of increasing sharpness as the control parameter $K$ is reduced to zero. On the other hand, the state $u=0$ is unstable, and solutions around this value vanish or coalesce over long time scales in a phenomenon known as metastability [28].

The interfacial properties of the Allen-Cahn equation in the fractional case have been previously analysed [19], indicating that for decreasing $\alpha$ the solution changes significantly faster near the center of the interface. Away from the centre the solutions become less steep and the whole interface becomes thicker, reflecting the non-local character of the fractional operator. However, the effect of fractional diffusion on the metastability of the solutions has still not been studied.

Figure 2 shows the effect of varying $\alpha$ in the metastability of solutions of the Allen-Cahn equation in $x \in[-1,1]$, with parameter $K=0.01$ and initial data $u(x, 0)=\frac{1}{2} \sin \left(\frac{3 \pi}{2} x\right)(\cos (\pi x)-1)$. For the pure diffusion case (Fig. 2(a)), the initial data evolves to an intermediate unstable equilibrium, followed by a rapid transition to a solution with just one interface. As the fractional power is decreased, the lifetime of the unstable interface is largely prolonged (Fig. 2(b)), eventually becoming fully stabilised due to the long-tailed influence of the fractional diffusion process (Fig. 2(c)). Our last Matlab example, Code 4, illus- 
trates the solution of the fractional-in-space Allen-Cahn equation using the proposed Fourier method.

\subsection{FitzHugh-Nagumo model-Excitable media}

The FitzHugh Nagumo model represents one of the simplest models for the study of excitable media $[29,30]$. The propagation of the transmembrane potential in the nerve axon is modeled by a diffusion equation with a cubic nonlinear reaction term, whereas the recovery of the slow variable is represented by a single ordinary differential equation in the form

$$
\begin{aligned}
\partial_{t} u & =-K_{u}(-\Delta)^{\alpha / 2} u+u(1-u)(u-a)-v \\
\partial_{t} v & =\epsilon(\beta u-\gamma v-\delta)
\end{aligned}
$$

where we consider the following choice of model parameters, $a=0.1, \epsilon=0.01$, $\beta=0.5, \gamma=1, \delta=0$, which is known to generate stable patterns in the system in the form of reentrant spiral waves. In our simulations, the trivial state $(u, v)=(0,0)$ was perturbed by setting the lower-left quarter of the domain to $u=1$ and the upper half part to $v=0.1$, which allows the initial condition to curve and rotate clockwise generating the spiral pattern. The domain is taken to be $[0,2.5]^{2}$, discretised using $N=256$ points in each spatial coordinate, with a diffusion coefficient $K_{u}=10^{-4}$.

Stable rotating solutions at $t=2000$ are presented in Figure 3 to illustrate the effect of fractional diffusion in the FitzHugh-Nagumo model. The width of the excitation wavefront (red areas) is markedly reduced for decreasing $\alpha$, so is the wavelength of the system, with the domain being able to accommodate a larger number of wavefronts for smaller $\alpha$.

However, it is important to emphasise here that the role of reducing the fractional power $\alpha$ is not equivalent to the influence of a decreased diffusion coefficient in the pure diffusion case (Figure 4). This can be clearly observed by comparison of Figures 3(b)-4(b) and Figures 3(c)-4(c): for approximately the same width of the excitation wavefront, the wavelength of the system is larger in the fractional diffusion case, due to the long-tailed mechanisms of the fractional Laplacian operator. These results are therefore consistent with those of Engler [31] for the fractional reaction diffusion Fisher equation, showing distinct effects of fractional diffusion to those of reduced conductivity for a family of travelling wave solutions. They also illustrate the use of fractional diffusion as a modelling tool to characterise intermediate dynamic states not solely described by pure diffusion mechanisms.

For different conductivities along each coordinate axis, a simple way to introduce anisotropy in the fractional-in-space reaction-diffusion model (5) is by means of expanding (4) in the form (see [21]):

$$
\partial_{t} u=-\sum_{i=1}^{N_{d}} K_{i}\left(-\frac{\partial^{2}}{\partial^{2} x_{i}}\right)^{\alpha_{i} / 2} u+f(u, t), \quad 1<\alpha_{i} \leq 2,
$$




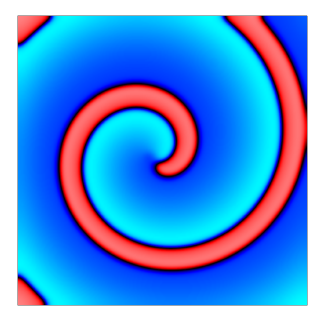

(a) $\alpha=2$

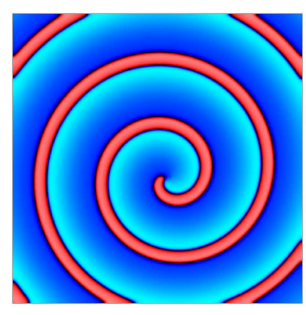

(b) $\alpha=1.7$

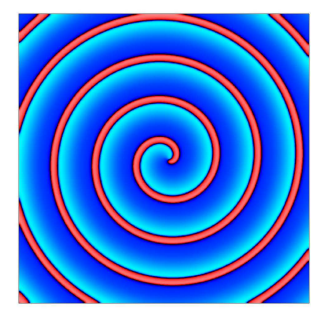

(c) $\alpha=1.5$

Figure 3: Spiral waves in the FitzHugh-Nagumo model for varying $\alpha$.

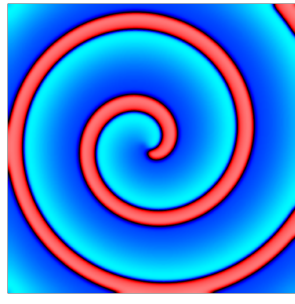

(a) $K_{u}=5 \times 10^{-5}$

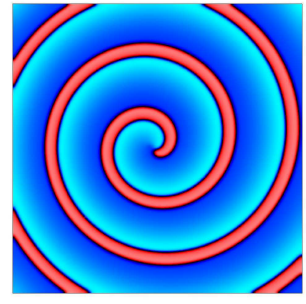

(b) $K_{u}=3 \times 10^{-5}$

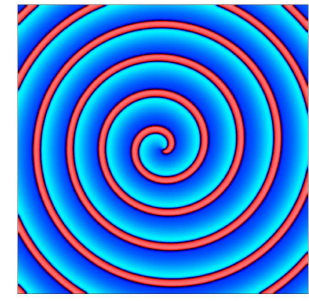

(c) $K_{u}=10^{-5}$

Figure 4: Solutions of the FitzHugh-Nagumo model for varying diffusion coefficient and $\alpha=2$.

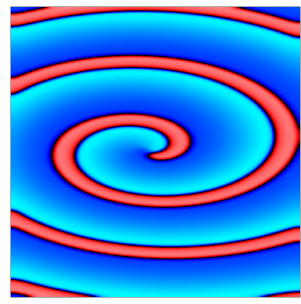

(a) $\frac{K_{y}}{K_{x}}=0.25, \frac{\alpha_{y}}{\alpha_{x}}=1$

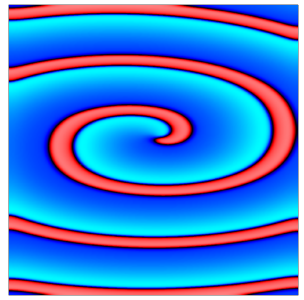

(b) $\frac{K_{y}}{K_{x}}=1, \frac{\alpha_{y}}{\alpha_{x}}=0.825$

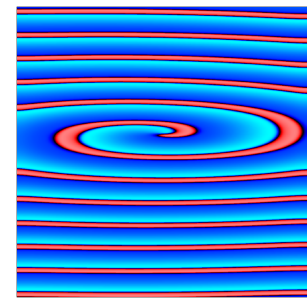

(c) $\frac{K_{y}}{K_{x}}=0.25, \frac{\alpha_{y}}{\alpha_{x}}=0.825$

Figure 5: Wave propagation in the FitzHugh-Nagumo model for different anisotropic ratios $\left(\alpha_{x}=2, K_{x}=K_{u}\right)$.

where $N_{d}$ is the spatial dimensionality of the problem, and with different fractional powers $\alpha_{i}$ in each spatial dimension. Examples of anisotropic propagation in the FitzHugh-Nagumo model, using a grid resolution of $400 \times 400$ points, are presented in Figure 5. For anisotropic diffusion ratios $K_{y} / K_{x}<1$, spiral waves now proceed following an elliptical pattern (Fig. 5(a)). Interestingly, anisotropic fractional ratios $\alpha_{y} / \alpha_{x}<1$ exert contrasted effects on the curvature of the solu- 
tions (Fig. 5(b)), reflecting distinct super-diffusion scales in each of the spatial dimensions of the system. The combination of the two sources of anisotropy yield a mixed effect of the two independent contributions (Fig. 5(c)), also further reducing the wavelength of the system, in agreement with the previous results illustrated by Figures 3 and 4 .

\subsection{Gray-Scott model-Morphogenesis}

The extension of the Fourier stencil to systems of reaction-diffusion equations is as well straightforward. We consider the fractional version of the Gray-Scott model $[32,33]$

$$
\begin{aligned}
& \partial_{t} u=-K_{u}(-\Delta)^{\alpha / 2} u-u v^{2}+F(1-u) \\
& \partial_{t} v=-K_{v}(-\Delta)^{\alpha / 2} v+u v^{2}-(F+\kappa) v,
\end{aligned}
$$

where $K_{u}, K_{v}, F$ and $\kappa$ are positive constants. For a ratio of diffusion coefficients $K_{u} / K_{v}>1$, the model is known to generate different mechanisms of pattern formation depending on the values of the feed, $F$, and depletion, $\kappa$, rates. Here we select $K_{u}=2 \times 10^{-5}, K_{u} / K_{v}=2, F=0.03$, and vary $\kappa$ in a range in which the standard diffusion model is known to exhibit interesting dynamics [34]. The domain of interest is taken to be $[0,1]^{2}$, discretised using $N=400$ points in each spatial coordinate. Initially, the entire system was placed in the trivial state $(u, v)=(1,0)$, and a $32 \times 32$ mesh point area located symmetrically about the centre of the grid was perturbed to $(u, v)=(1 / 2,1 / 4)$. The initial disturbance then propagates outward from the central square until the entire grid is affected by the initial perturbation.

Figure 6 summarizes the effects of fractional diffusion in the Gray-Scott model. For $\kappa=0.055$ (Fig. 6(a)), the model with standard diffusion $(\alpha=2)$ is known to organize in a steady state field of negative solitons. A reduction in the fractional order of the diffusive process $(\alpha=1.7)$ produces a decrease in the velocity of propagation of the initial perturbation, and a much finer granulation in the size of the structures of the final steady state field. For smaller values of the fractional power $(\alpha=1.5)$, a new process of nucleation of structures in the centre and in the boundaries of the domain is observed, that then grow outward until the entire domain reaches its final steady state configuration.

For $\kappa=0.061$ (Fig. 6(b)), the original model produces a wavefront propagation partially driven by curvature, and a final steady state pattern showing the presence of filaments. The curvature driven mechanisms are increased by the diffusive effects of the fractional Laplacian operator, yielding a final field formed by much thiner filaments, and steady state patterns totally different to those generated by standard diffusion.

Finally, the Gray-Scott model exhibits mitosis for $\kappa=0.063$ under conditions of normal diffusion (Fig. 6(c)). However, the replication pattern is completely altered when the fractional order of the model is decreased, as shown in this Figure for $\alpha=1.7$. In fact, further reductions of the fractional power, as shown for $\alpha=1.5$, produce dynamical states where solitons and filaments may 

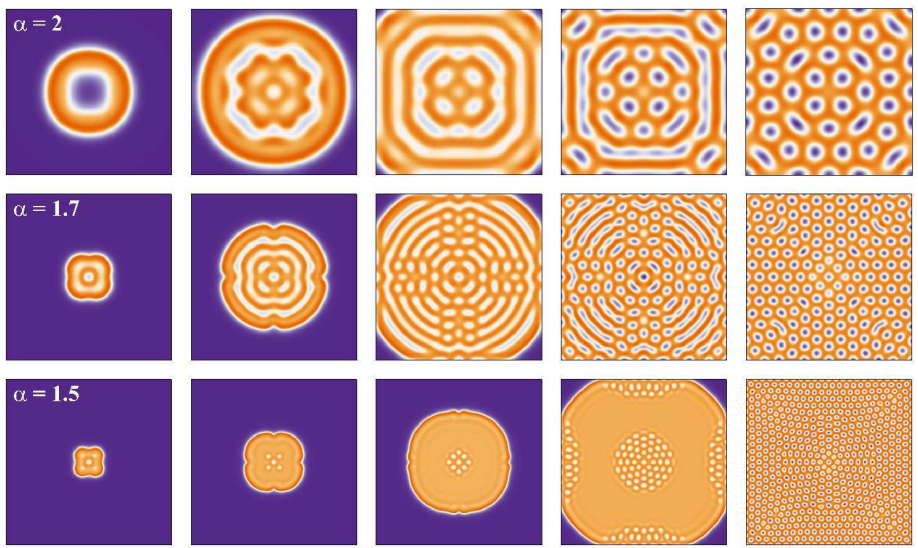

(a) $\kappa=0.055$
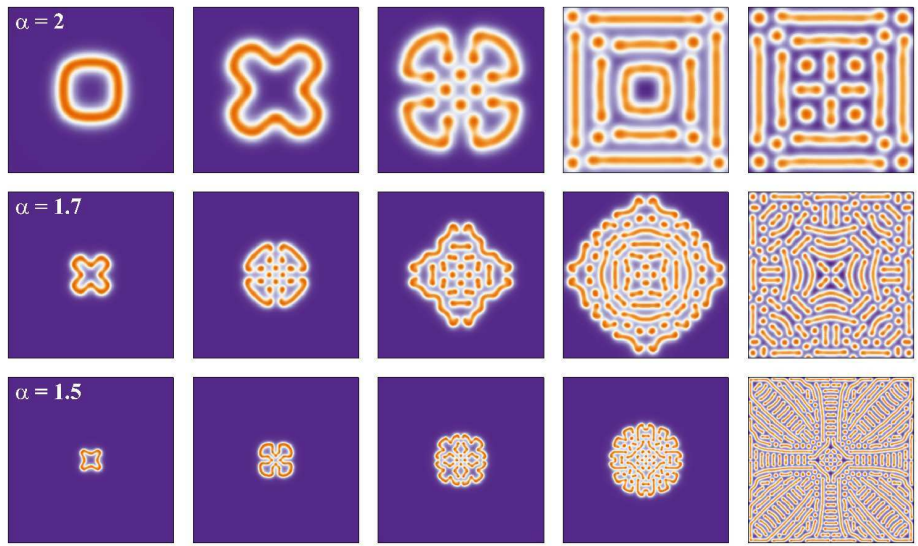

(b) $\kappa=0.061$
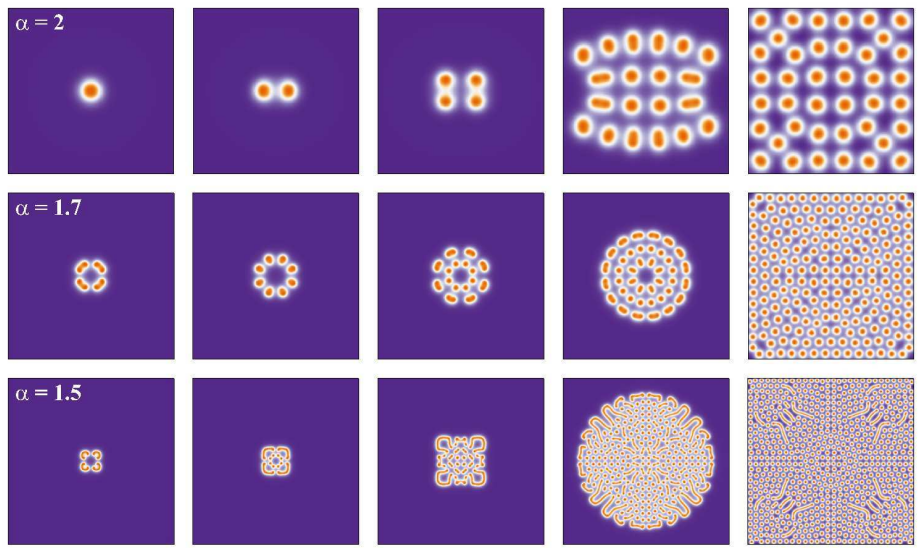

(c) $\kappa=0.063$

Figure 6: Pattern formation in the Gray-Scott model for different values of parameter $\kappa$ and fractional power $\alpha$. 

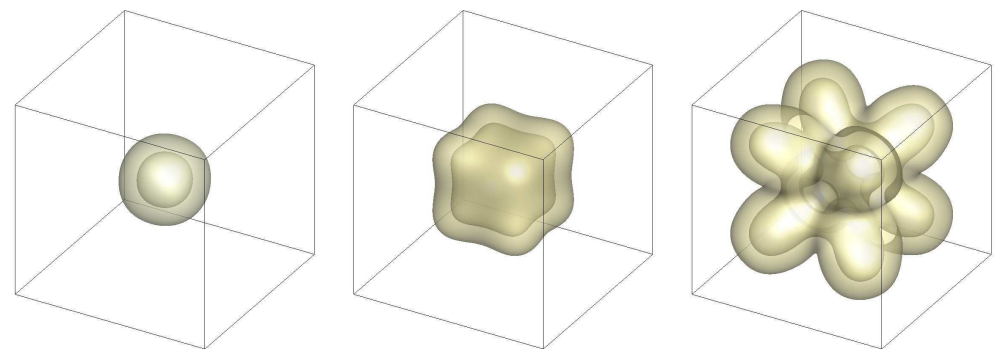

(a) $\alpha=2$
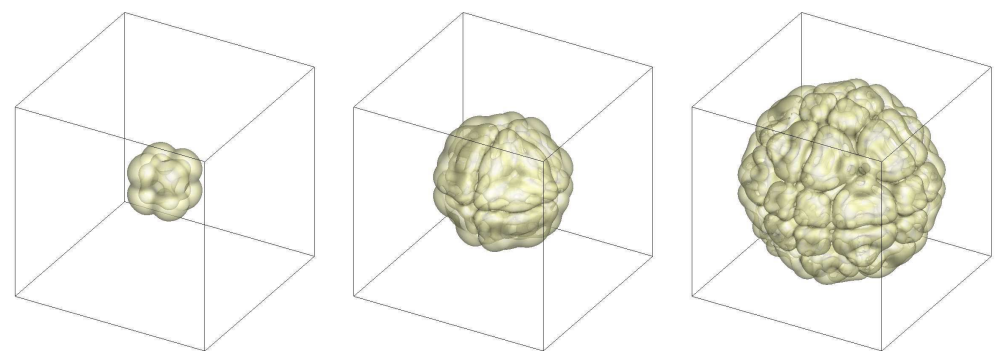

(b) $\alpha=1.5$

Figure 7: Isosurfaces $u=0.65$ for the Gray-Scott model for $\kappa=0.061$.

coexist, the latter slowing self-dividing into the former until the whole domain is filled by a soliton-like pattern.

\subsection{Gray-Scott model - Patterns in Three Dimensional Space}

Numerical simulation of the Gray-Scott model in three spatial dimensions represents an even more interesting scenario where more exotic patterns may arise. For the parameter regime where wavefront motion is partially driven by curvature $(\kappa=0.061)$, and for two different $\alpha$, Figure 7 illustrates the three dimensional propagation of the initial perturbation before the interaction of the solution with the domain boundaries. As can be clearly appreciated, the smooth growing of lobes in the presence of normal diffusion (Fig. 7(a)) is replaced by more intriguing patterns in the fractional diffusion case (Fig. 7(b)). For improvement of visualization results, the domain size is $[0,1]^{3}$ in Fig. $7(\mathrm{a})$, and $[0.25,0.75]^{3}$ in Fig. $7(\mathrm{~b})$.

\section{Conclusions}

In this paper, Fourier spectral methods have been introduced as an attractive and easy-to-code alternative for the integration of fractional-in-space reactiondiffusion equations. These methods offer several advantages over traditional alternatives. Since the operator is non-local, the benefits of using a basis with locally supported elements are destroyed. Hence, the use of an orthogonal, with 
respect to the operator, non-local basis is preferable and will give rise to a fully diagonal representation of the fractional operator, thus avoiding the solution of large systems of equations or the use of matrix transfer techniques. In terms of accuracy and efficiency, Fourier methods have been proven to be not only advantageous relative to memory requirements (number of discretisation points) when compared to low-order schemes, but also computationally efficient in execution times. Furthermore, the use of established discrete fast Fourier transforms ensures efficiency, makes immediate the implementation of appropriate boundary conditions, and allows the extension of the stencil to two and three dimensions in a completely straightforward manner.

Simulation results of the fractional-in-space Allen-Cahn, FitzHugh-Nagumo and in particular Gray-Scott models show that such problems can have very different dynamics to standard diffusion, and as such represent a powerful modelling approach for understanding the many aspects of heterogeneity.

Despite their higher order of convergence, the biggest drawback of spectral methods is their inability to handle irregularly shaped domains, since the domain of interest must be simple enough to allow the use of an appropriate series of polynomial or trigonometric basis functions in where to expand the global highorder interpolants. However, recent work in combining spectral methods with domain embedding techniques has allowed the extension of these methods to irregular domains $[27,35,36,37,38]$. The use of these techniques may constitute a suitable approach for extending our results in the fractional-in-space setting to irregular shape geometries.

The applications presented in this paper also have illustrated that Fourier spectral methods can easily handle anisotropy when this is constant throughout the entire integration domain. For spatially-dependent conductivities, the representation of the fractional operator in Fourier space would be the convolution of the Fourier coefficients of such conductivities and those of the fractional Laplacian, thus losing the diagonal structure of the operator and requiring the solution of full systems of equations. However, due to the smaller number of degrees of freedom required by Fourier methods to achieve a given accuracy, they could still be competitive when compared to low order schemes. On the other hand, the use of spectral differentiation matrices [28, 39], in combination with the matrix transfer technique, could also be helpful in order to overcome these limitations.

Finally, an additional advantage of finite element and finite difference methods is their ability to accommodate adaptive mesh refinement for non-smooth solutions. Rather than the use of mesh refinement algorithms, the easiest way to incorporate spatial adaptivity in spectral methods seems to be by means of the so-called moving mesh techniques, also known as $r$-adaptivity [40, 41]. The implementation of these techniques for fractional-in-space reaction-diffusion equations may also constitute another of our future lines of research. 


\section{Acknowledgments}

This publication is based on work supported by Award No. KUK-C1-013-04, made by King Abdullah University of Science and Technology (KAUST).

\section{References}

[1] E. E. Adams, L. W. Gelhar, Field study of dispersion in a heterogeneous aquifer: 2. Spatial moment analysis, Water Resources Res. 28 (1992) 32933307.

[2] D. A. Benson, S. Wheatcraft, M. M. Meerschaert, Application of a fractional advection-dispersion equation, Water Resources Res. 36 (2000) 14031412 .

[3] M. M. Meerschaert, D. A. Benson, S. W. Wheatcraft, Subordinated advection-dispersion equation for contaminant transport, Water Resource Res. 37 (2001) 1543-1550.

[4] E. Scalas, R. Gorenflo, F. Mainardi, Fractional calculus and continuous time finance, Physica A 284 (2000) 376-384.

[5] R. L. Magin, O. Abdullah, D. Baleanu, X. J. Zhou, Anomalous diffusion expressed through fractional order differential operators in the Bloch-Torrey equation, J. of Mag. Res. 190 (2008) 255-270.

[6] P. Becker-Kern, M. M. Meerschaert, H. P. Scheffler, Limit theorem for continuous time random walks with two time scales, J. App. Prob. 41 (2004) $455-466$.

[7] R. Metzler, J. Klafter, The random walk's guide to anomalous diffusion: A fractional dynamics approach, Phys. Rep. 339 (2000) 1-77.

[8] Y. Zhang, D. A. Benson, D. M. Reeves, Time and space nonlocalities underlying fractional-derivative models: Distinction and literature review of field applications, Adv. in Water Res. 32 (2009) 561-581.

[9] M. M. Meerschaert, J. Mortensenb, S. W. Wheatcraft, Fractional vector calculus for fractional advection-dispersion, Physica A 367 (2006) 181-190.

[10] M. Riesz, L'intégrale de Riemann-Liouville et le problème de Cauchy, Acta Mathematica 81 (1949) 1-222.

[11] J. P. Bouchaud, A. Georges, Anomalous diffusion in disordered media: Statistical mechanisms, models and physical applications, Phy. Rep. 195 (1990) 127-293.

[12] I. Turner, M. Ilić, P. Perr, The use of fractional-in-space diffusion equations for describing microscale diffusion in porous media, in: 11th International Drying Conference, Magdeburg, Germany, 2010. 
[13] M. M. Meerschaert, C. Tadjeran, Finite difference approximations for twosided space-fractional partial differential equations, App. Num. Math 56 (2006) 80-90.

[14] J. Roop, Computational aspects of FEM approximations of fractional advection dispersion equations on bounded domains on $R^{2}$, J. Comp. Appl. Math. 193 (2005) 243-268.

[15] M. Ilić, F. Liu, I. Turner, V. Anh, Numerical approximation of a fractionalin-space diffusion equation, I. Frac. Calc. and App. Anal. 8 (2005) 323-341.

[16] F. Liu, P. Zhuang, V. Anh, I. Turner, K. Burrage, Stability and convergence of the finite difference method for the space-time fractional advectiondiffusion equation, App. Num. and Comp. 91 (2007) 12-20.

[17] H.-K. Pang, H.-W. Sun, Multigrid method for fractional diffusion, J. Comp. Phys. 231 (2012) 693-703.

[18] Q. Yang, I. Turner, F. Liu, M. Ilić, Novel numerical methods for solving the time-space fractional diffusion equation in 2D, SIAM J. Sci. Comp. 33 (2011) 1159-1180.

[19] K. Burrage, N. Hale, D. Kay, An efficient implicit FEM scheme for fractional-in-space reaction-diffusion equations.

[20] Q. Yang, F. Liu, I. Turner, Numerical methods for fractional partial differential equations with Riesz space fractional derivatives, App. Num. Mod. 34 (2010) 200-218.

[21] K. Burrage, D. Kay, Efficient numerical solvers for fractional diffusion, In Review.

[22] X. Li, C. Xu, Existence and uniqueness of the weak solution of the spacetime fractional diffusion equation and a spectral method approximation, Commun. Comput. Phys. 8 (2010) 1016-1051.

[23] M. M. Khader, On the numerical solutions for the fractional diffusion equation, Commun. Nonlinear Sci. Numer. Simulat. 16 (2010) 2535-2542.

[24] X. Li, C. Xu, A space-time spectral method for the time fractional diffusion equation, SIAM J. Numer. Anal. 47 (2009) 2108-2131.

[25] E. Hanert, A comparison of three eulerian numerical methods for fractionalorder transport models, Environ. Fluid Mech. 10 (2010) 7-20.

[26] W. L. Briggs, V. E. Henson, The DFT: an owner's manual for the discrete Fourier transform, SIAM, Philadelphia, 2000.

[27] A. Bueno-Orovio, V. M. Pérez-García, Spectral smoothed boundary methods: The role of external boundary conditions, Numer. Meth. Part. Differ. Equ. 22 (2006) 435-448. 
[28] L. N. Trefethen, Spectral methods in Matlab, SIAM, Philadelphia, 2000.

[29] R. FitzHugh, Impulses and physiological states in theoretical models of nerve membranes, Biophys. J. 1 (1961) 445-466.

[30] J. Nagumo, S. Animoto, S. Yoshizawa, An active pulse transmission line simulating nerve axon, Proc. Inst. Radio Engineers 50 (1962) 2061-2070.

[31] H. Engler, On the speed of spread for fractional reaction-diffusion equations, Int. J. Diff. Eqn.doi:10.1155/2010/315421.

[32] P. Gray, S. K. Scott, Autocatalytic reactions in the isothermal, continuous stirred tank reactor: isolas and other forms of multistability, Chem. Eng. Sci. 38 (1983) 29-43.

[33] P. Gray, S. K. Scott, Sustained oscillations and other exotic patterns of behavior in isothermal reactions, J. Phys. Chem. 89 (1985) 22-32.

[34] J. E. Pearson, Complex patterns in a simple system, Science 261 (1993) 189-192.

[35] A. Bueno-Orovio, Fourier embedded domain methods: periodic and $C^{\infty}$ extension of a function defined on an irregular region to a rectangle via convolution with Gaussian kernels, App. Math. Comp. 183 (2006) 813-818.

[36] A. Bueno-Orovio, V. M. Pérez-García, F. H. Fenton, Spectral methods for partial differential equations in irregular domains: The spectral smoothed boundary method, SIAM J. Sci. Comput. 28 (2006) 886-900.

[37] S. H. Lui, Spectral domain embedding for elliptic PDEs in complex domains, J. Comput. Appl. Math. 225 (2009) 541-557.

[38] F. Sabetghadam, S. Sharafatmandjoor, F. Norouzi, Fourier spectral embedded boundary solution of the Poisson's and Laplace equations with Dirichlet boundary conditions, J. Comput. Phys. 228 (2009) 55-74.

[39] J. A. C. Weideman, S. C. Reddy, A matlab differentiation matrix suite, ACM Trans. Math. Softw. 26 (2000) 465-519.

[40] W. M. Feng, P. Yu, S. Y. Hu, Z. K. Liu, Q. Du, L. Q. Chen, Spectral implementation of an adaptive moving mesh method for phase-field equations, J. Comput. Phys. 220 (2006) 498-510.

[41] L. S. Mulholland, W. Z. Huang, D. M. Sloan, Pseudospectral solution of near-singular problems using numerical coordinate transformations based on adaptivity, SIAM J. Sci. Comput. 19 (1998) 1261-1289. 



\section{RECENT REPORTS}

12/26 Age related changes in speed and mechanism of adult skeletal muscle stem cell migration

Collins-Hooper

Woolley

Dyson

Patel

Potter

Baker

Gaffney

Maini

Dash

Patel

12/27 The interplay between tissue growth and scaffold degradation in engineered tissue constructs

ODea

Osborne

El Haj

Byrne

Waters

12/28 Non-linear effects on Turing patterns: time oscillations and Aragon chaos.

Barrio

Woolley

Baker

Maini

12/29 Colorectal Cancer Through Simulation and Experiment

Kershaw

Byrne

Gavaghan

Osborne

12/30 A theoretical investigation of the effect of proliferation and adhesion on monoclonal conversion in the colonic crypt

Mirams

Fletcher

Maini

Byrne

12/31 Convergent evolution of spiny mollusk shells points to elastic energy minimum

Chirat

Moulton

Shipman

Goriely

12/32 Three-dimensional oblique water-entry problems at small dead-

Moore rise angles

Howison

Ockendon

Oliver

12/33 Second weak order explicit stabilized methods for stiff stochastic

Abdulle differential equations

Vilmart

Zygalakis

12/34 The sensitivity of Graphene 'Snap-through' to substrate geometry Wagner

Vella

Peppin

Style

12/35 The physics of frost heave and ice-lens growth

Sprittles

Shikhmurzaev face Formation Process

Sprittles

12/37 The Dynamics of Liquid Drops and their Interaction with Solids of Shikhmurzaev 
12/40 A Branch and Bound Algorithm for the Global Optimization of Hessian Lipschitz Continuous Functions

12/41 The Orthogonal Gradients Method: a Radial Basis Functions

Piret Method for Solving Partial Differential Equations on Arbitrary Surfaces

12/42 Squeeze-Film Flow in the Presence of a Thin Porous Bed, with Application to the Human Knee Joint

Knox

Wilson

Duffy

McKee

12/43 Gravity-driven draining of a thin rivulet with constant width down a slowly varying substrate

Paterson

Wilson

Duffy

12/44 The 'Sticky Elastica': Delamination blisters beyond small deformations

Wagner

Vella

12/45 Stochastic models of intracellular transport

Bressloff

Newby

12/46 The effects of noise on binocular rivalry waves: a stochastic neural field model

Webber

Bressloff

12/47 An Ensemble Bayesian Filter for State Estimation

Farmer

12/48 Simulation of cell movement through evolving environment: a fictitious domain approach

Séguis

Burrage

Erban

Kay

12/49 The Mathematics of Liquid Crystals: Analysis, Computation and

Majumdar Applications

Copies of these, and any other OCCAM reports can be obtained from:

Oxford Centre for Collaborative Applied Mathematics

Mathematical Institute

24 - 29 St Giles'

Oxford

OX1 3LB

England

www.maths.ox.ac.uk/occam 